\title{
Scaled Silicon Nanoelectromechanical (NEM) Hybrid Systems
}

\author{
Hiroshi Mizuta $^{1}$ *, Mario A. Garcia-Ramirez ${ }^{1}$, Faezeh. A. Hassani ${ }^{1}$, Mohammad. A. Ghiass ${ }^{1}$, \\ Nima Kalhor ${ }^{1}$, Zakaria Moktadir ${ }^{1}$, Yoshishige Tsuchiya ${ }^{1}$, Shunichiro Sawai ${ }^{2}$, Jun Ogi ${ }^{2}$, Shunri Oda ${ }^{2}$ \\ ${ }^{1}$ NANO Group, School of Electronics and Computer Science, University of Southampton, Southampton, UK \\ ${ }^{2}$ Quantum Nanoelectronics Research Center, Tokyo Institute of Technology, Tokyo, Japan \\ * Email: hm2@ecs.soton.ac.uk
}

\begin{abstract}
In this paper we overview recent attempts at co-integrating silicon nano-electro-mechanical systems (NEMS) with nanoelectronic devices aiming to add more functionalities to conventional electronic devices in 'More-than-Moore' domain and also explore novel operating principles in 'Beyond CMOS' domain.
\end{abstract}

\section{Co-integration of NEMS and MOSFETs for 'More-than-Moore' applications}

Silicon VLSI technology developed and matured over the past decades has been fully exploited to build the vast technology area of micro-electromechanical systems (MEMS). Along with a rapid expansion of the MEMS market, there have also been continuous efforts at making the MEMS smaller (Fig. 1) in order to boost the operating frequency to $\mathrm{GHz}$ and beyond. The appearance of high-speed nano-electromechanical systems (NEMS) is tempting enough for us to consider the hybridization of the NEMS and conventional silicon electronic devices ('More than Moore') because we expect such hybrid systems enhance scaling of functional density \& performance while simultaneously reducing the power dissipation beyond the conventional CMOS-based systems.

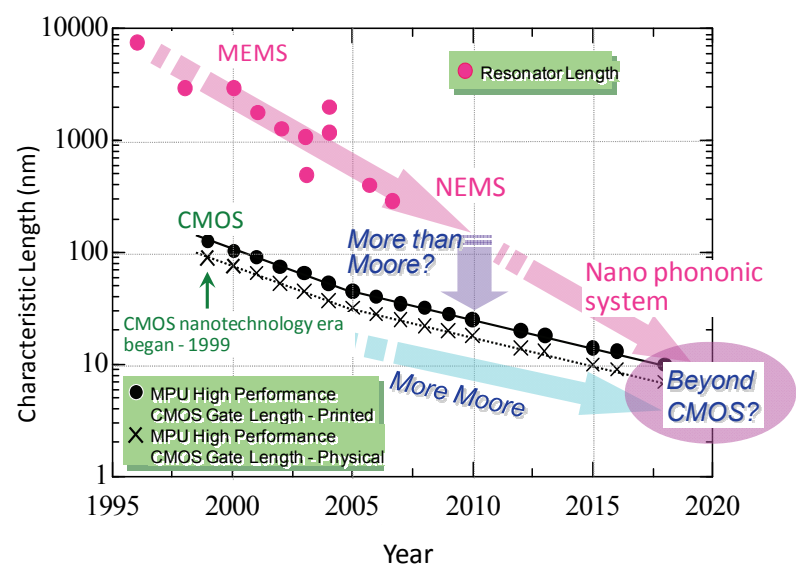

Figure 1 Recent trend of MEMS/NEMS downscaling along with CMOS miniaturization.
A variety of new hybrid NEM-CMOS devices have recently been studied for advanced switch, memory and sensing applications (see Table 1). A pioneering hybrid device is a suspended-gate (SG) FET [1] which features a movable gate electrode located on a conventional oxide / silicon substrate via an air gap. Thanks to unique electro-mechanical pull-in / pull-out operations, the SGFETs exhibit very abrupt electrical switching with a subthreshold swing much smaller than a theoretical limit of $60 \mathrm{mV} / \mathrm{dec}$ for conventional MOSFETs as well as extremely low off current. The SGFETs therefore attract much attention in particular for power management applications. As for the memory applications, two types of novel high-speed and nonvolatile NEM-MOS hybrid memory devices have been proposed in order to realize nonvolatile RAM. The bistable floating gate (FG) NEM memory [2] was first proposed, which features a self-buckling $\mathrm{SiO}_{2}$ floating gate (FG) with embedded $\mathrm{Si}$ nanodots as single-electron storage. The self-buckling FG is flip-flopped electrically with the gate voltage, and the bistable mechanical states are detected with a readout MOSFET located underneath.

Table 1 Various NEM-MOS hybrid functional devices

\begin{tabular}{|c|c|c|}
\hline$\frac{c}{\frac{c}{u}}$ & 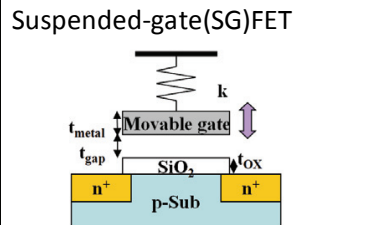 & $\begin{array}{l}\text { - Abrupt switching } \\
\text { with } S<60 \mathrm{mV} / \mathrm{dec} \\
\text { - Very low leakage } \\
\text { - Power management } \\
\text { applications }\end{array}$ \\
\hline 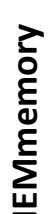 & $\begin{array}{l}\text { g FG Memory } \\
\text { dot Memory } \\
\text { ded-gate }(S G)\end{array}$ & $\begin{array}{l}\text { - Seriously nonvolatile } \\
\text { - Fast write/erase } \\
\text { operation } \\
\text { - Conventional silicon } \\
\text { process compatible }\end{array}$ \\
\hline 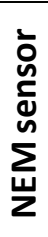 & $\begin{array}{l}\text { - Vibrating body FET } \\
\text { - Resonant SG MOSFET }\end{array}$ & $\begin{array}{l}\text { Extremely high mass } \\
\text { responsibity of } \\
\text { zeptogram/Hz order } \\
\text { Co-integration with } \\
\text { SOI-MOSFET } \\
\text { circuits }\end{array}$ \\
\hline
\end{tabular}


The other one is a suspended gate (SG) $\mathrm{Si}$ nanodot memory (SGSNM) [3] in which the SG is pulled in to the FG only when we programme and erase the information. Recent theoretical study shows that the SGSNM achieves programme/erase times of few nanoseconds with serious nonvolatility. As for the sensing applications, a new in-plane resonant NEM sensor [4] has been proposed based on a mass-detection principle. This nanosensing device features a silicon resonant-suspended-gate and an in-plane MOSFET co-integrated on an ultrathin SOI platform and enables sub-attogram-level mass detection. Despite a number of potential advantages demonstrated for experimental NEM-MOS hybrid devices, there still remain various crucial issues to be clarified, in particular, scalability which takes the influences of nanoscopic Van der Waals force and Casimir effect into consideration [5][6].

\section{NEM-SET hybrid devices and atomically-scaled $\mathrm{Si}$ NEM systems for 'Beyond-CMOS' domain}

By downscaling the NEMS towards a 100-nm-regime and even smaller, we may explore a novel hybrid system of NEMS and single-electron transistors (SETs). There are a number of new phenomena associated with strong coupling of single-electron tunnelling and low-dimensional phonons, such as phonon blockade [7], single-electron quantum shuttle [8] and the quantum ground state of a mechanical resonator [9]. Manipulation of nanophononic states in scaled silicon nanostructures may be exploited to develop a novel approach to thermal management and energy transfer interaction in 'Beyond CMOS' information processing.

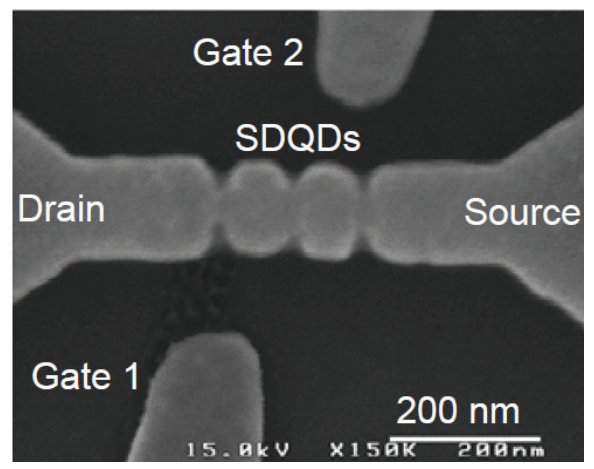

Figure 2 The SEM image of fabricated SDQDs structure. The lateral dimension of DQDs is about $150 \mathrm{~nm}$.

A suspended quantum dot (QD) SET [10] is particularly attractive to investigate the interaction between single electrons and low-dimensional phonons in the NEM structures. Figure 2 shows suspended double quantum dots (SDQDs) built on a doubly-clamped $\mathrm{Si}$ beam with two side gates. The SDQD SET was fabricated on 40-nm-thick heavily-P-doped $\left(2 \times 10^{19} \mathrm{~cm}^{-3}\right)$ silicon-on-insulator (SOI) substrate by using EB lithography [11]. Bias voltages applied to the two side gates, $V_{g 1}$ and $V_{g 2}$, control the electro-chemical potentials of the individual DQDs. A single-electron tunnels through DQDs elastically when the chemical potentials align.

Figure 3 shows the drain current measured at $120 \mathrm{mK}$ with $V_{d s}=200 \mu \mathrm{V}$ as a function of $V_{g 1}$ and $V_{g 2}$. The tunnel current is enhanced around the anti-crossing points of two charging lines, resulting in a pair of bias triangles. Fine structures submerged in the bias triangles were recently clarified by decomposing a broad current peak across the bias triangle into a few current peaks.

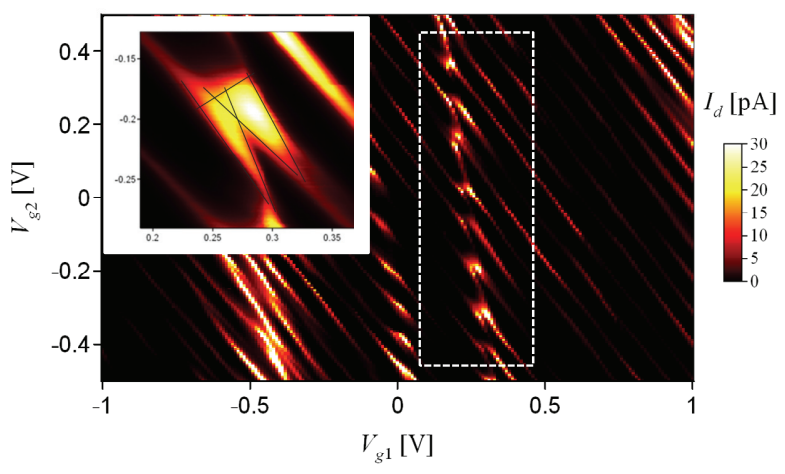

Figure 3 Drain-to-source current measured at $120 \mathrm{mK}$ with $\mathrm{Vds}=200 \mu \mathrm{V}$ as a function of $\mathrm{Vg} 1$ and $\mathrm{Vg} 2$. The typical characteristics for serial DQDs were observed in the region shown by a broken-line rectangular. The inset shows a pair of bias triangles observed around one of the anti-crossing regions.

In Fig. 4(a), the largest peak centered at $\Delta E=0$ corresponds to elastic (coherent) tunneling, and the peaks centered at $\Delta E>0$ correspond to inelastic tunneling of electrons with phonon emission. By comparing these experimental results with the phonon spectral density calculated for the SDQDs by using the continuum model (Fig. 4(b)), it was found that the observed current peals were attributable to the enhanced interaction between the electrons confined in the SDQDs and dilatational- and flexural-mode phonons of the suspended Si beam.

A good agreement between the experimental and theoretical phonon peak energies enables us to understand and even manipulate fundamental energy dissipation processes of tunnelling electrons in Si NEM structures. For example, the peak energy in the spectral density associated with the van Hove singularities of dilatational mode can be controlled via Si slab thickness as shown in Fig. 5: the peak energy increases approximately inverse proportional to the $\mathrm{Si}$ slab thickness. 

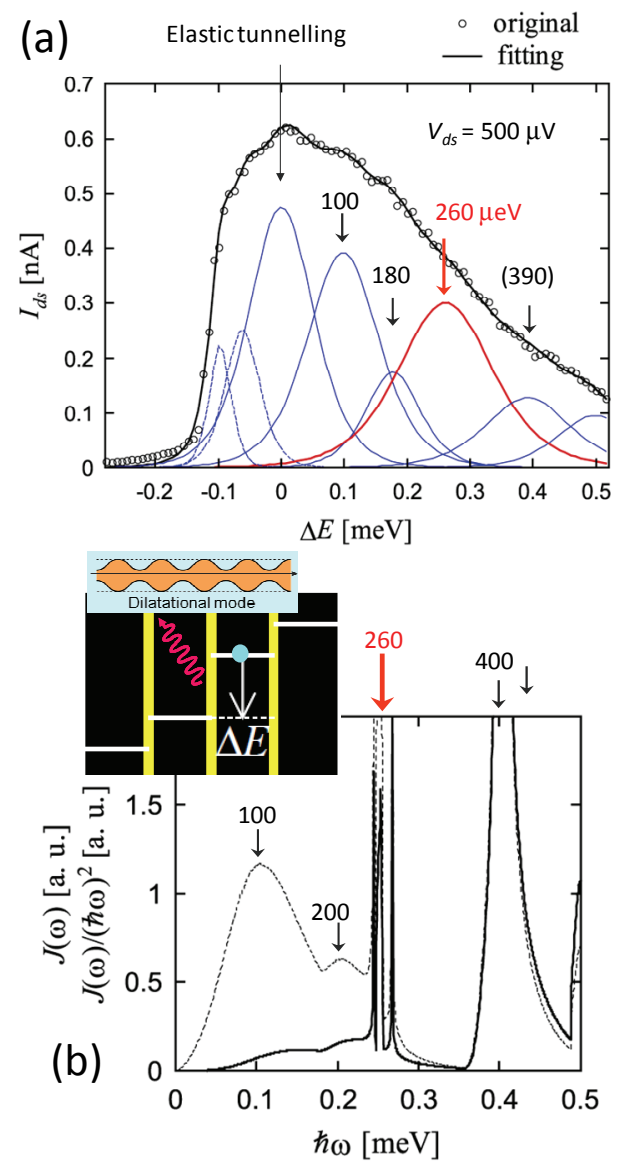

Figure 4 (a) $I_{\mathrm{ds}}$ versus the energy difference $\Delta E$ between the double QDs with $V_{\mathrm{ds}}=500 \mu \mathrm{V}$. The original data (circle) are fitted by a sum of peaks. (b) The calculated phonon spectral density (solid line) and its ratio to the square of the phonon energy (dotted line). The arrow shows the van Hove singularities of the dilatational mode that is schematically shown in the inset.

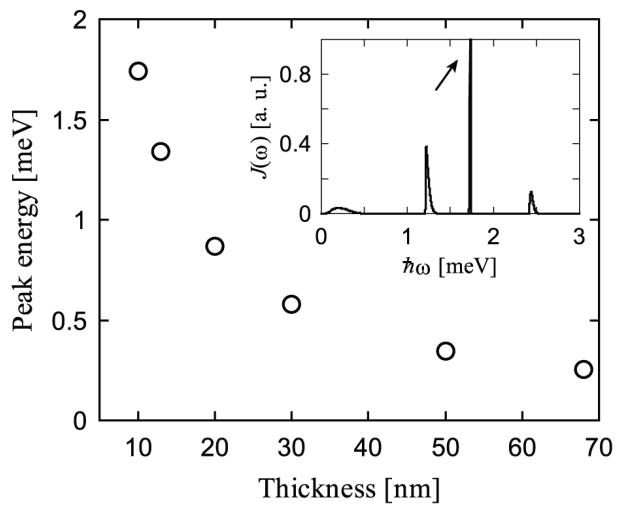

Figure 5 Peak phonon energy calculated for one of dilatational modes as a function of $\mathrm{Si}$ slab thickness. The inset shows phonon spectral density for a 10-nm-thick Si slab.
Extremely-scaled Si NEMS will eventually exploit electro-mechanical properties of atomically-controlled $\mathrm{Si}$ nanostructures. Ab-initio simulation of 'nanophonons' has recently been conducted for the H-terminated ultrathin $\mathrm{Si}$ films of from 3 to 10 atomic layers in thickness [12]. Figure 6 shows the dispersion relationship calculated for the 5-atomic-layer-thick film in comparison with those for bulk $\mathrm{Si}$. The phonon bandgap is observed both in the [1 -110$]$ and [ $\left[\begin{array}{lll}1 & 1 & 0\end{array}\right]$ directions which is defined between the energy maximum of the acoustic branches and the energy minimum of the right above optical-like branch. The formation of the phonon bandgaps are closely related to the $\operatorname{Si}(001) 2 \times 1$ surface dimers which line up in the [1 -1

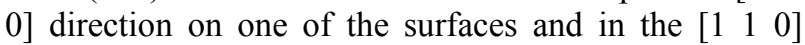
direction on the other surface. In contrast to the bulk Si atoms, the surface $\mathrm{Si}$ atoms are covalently-bound alternately in the direction and therefore hold the structural and mechanical periodicity of twice as long as that for bulk Si.
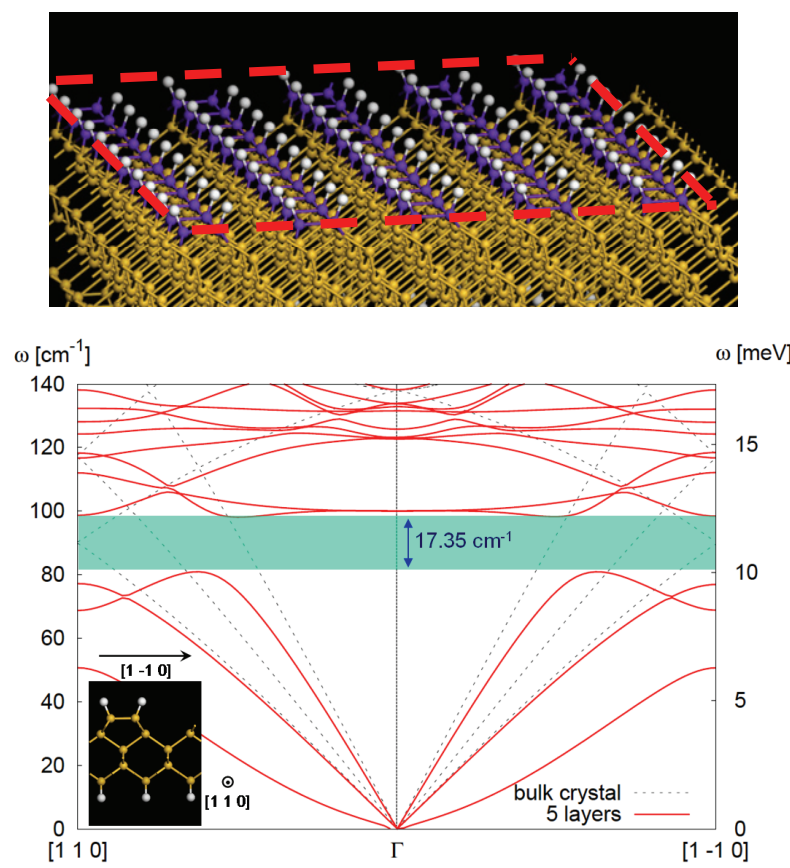

Figure 6 Ab-initio simulation of nanophononic spectra for a hydrogen-terminated ultrathin $\mathrm{Si}$ film of 5 atomic layers in thickness. A hatched region shows a phonon bandgap formed both in the (110) and (1-10) directions.

The magnitude of the bandgaps decreases with increasing the film thickness and finally vanishes for the thickness above 8 atomic layers. This is because the impact of the surface dimers on the entire dispersion relationships is reduced relative to that of the bulk $\mathrm{Si}$ atoms. Those nanophononic properties are quite different from those for bulk silicon and may be exploited as one 
of approaches to nanoscale thermal management and novel energy transfer interactions. In addition, such periodic nanophononic structures may also work as photonic crystals, referred as phoXonic crystals, and offer a possibility of co-engineering phonons and photons [13], leading to novel nano opto-acoustic device applications.

\section{Acknowledgments}

The authors wish to acknowledge the partial support by SORST JST (Japan Science and Technology) and MEXT KAKENHI 18310097 and 16206030 Japan.

\section{References}

[1] N Abelé et al., IEDM Techn. Dig. 2005, 1075 (2005)

[2] Y. Tsuchiya et al., J. Appl. Phys 100, 094306 (2006).

[3] M. A. Garcia-Ramirez et al., Microelectronics Engineering 87, 1284 (2010).

[4] F. A. Hassani, et al., Ext Abstract of the 2009 International Conference on Solid State Devices and Materials, 1332 (2009).

[5] F. Michael Serry et al., J. Appl. Phys. 84, 2501, (1998).

[6] E. Buks and M. L. Roukes, Phys. Rev. B 63, 0 33402 (2001)

[7] E. M. Weig et al., PRL 92, 046804 (2004).

[8] A. Erbe et al., PRL 87, 096106 (2001).

[9] A. D. O'Connel et al., Nature 464, 697 (2010).

[10] J. Ogi et al., Jpn. J. Appl. Phys. 49, 044001 (2010)

[11] J. Ogi et al., Jpn. J. Appl. Phys. 49, 045203 (2010)

[12] S. Sawai et al., IEEE Silicon Nanoelectronics Workshop, M0200 (2008)

[13] M. Eichenfield et al., Nature 462, 78 (2009) 